Pacific Journal of Mathematics

AN ADDITION FORMULA FOR THE INDEX OF SEMIGROUPS
OF ENDOMORPISMS OF $B(H)$ 


\title{
AN ADDITION FORMULA FOR THE INDEX OF SEMIGROUPS OF ENDOMORPHISMS OF $B(H)$
}

\author{
William ARVESON
}

To the memory of Henry Dye: teacher, colleague, friend

It is shown that the numerical index $d_{*}(\alpha)$ of a semigroup $\alpha$ of *-endomorphisms of $B(H)$ obeys the logarithmic addition formula

$$
d_{*}(\alpha \otimes \beta)=d_{*}(\alpha)+d_{*}(\beta) .
$$

The proof makes essential use of the theory of continuous product systems

1. Introduction. Let $\alpha=\left\{\alpha_{t}: t \geq 0\right\}$ be a semigroup of normal *-endomorphisms of $B(H)$ such that $\alpha_{t}(1)=1$ and $\left\langle\alpha_{t}(A) \xi, \eta\right\rangle$ is continuous in $t$ for fixed $\xi, \eta \in H$ and $A \in B(H)$. Following Powers [2] we shall refer to such an $\alpha$ as an $E_{0}$-semigroup. It will be convenient to rule out the degenerate case in which the $\alpha_{t}$ 's are all automorphisms, and hence we require that $\alpha_{t}(B(H)) \neq B(H)$ for some (and therefore every) positive $t$. On the other hand, we will occasionally need to drop the hypothesis that $\alpha_{t}(1)=1$, and will refer to such an $\alpha$ simply as a *-semigroup. We emphasize that it is essential for the techniques below that all Hilbert spaces be separable.

In [1], a numerical invariant $d_{*}(\alpha)$ was introduced for $E_{0}$-semigroups $\alpha$ which can be defined rather concretely as follows. Fixing $\alpha$, let $\mathscr{U}_{\alpha}$ denote the set of all strongly continuous semigroups $U=\left\{U_{t}: t \geq 0\right\}$ of bounded operators on the Hilbert space $H$ of $\alpha$ satisfying $U_{0}=1$ and

$$
\alpha_{t}(A) U_{t}=U_{t} A, \quad A \in B(H), t \geq 0 .
$$

$\mathscr{U}_{\alpha}$ can be empty (cf. [3]). But if it is not, then for every pair of elements $U, V \in \mathscr{U}_{\alpha}$, there is a unique complex number $c(U, V)$ such that

$$
V_{t}^{*} U_{t}=e^{t c(U, V)} 1, \quad t \geq 0 .
$$

The function $c$ is self-adjoint $(\overline{c(U, V)}=c(V, U))$ and is conditionally positive definite in the sense that for every finite set of complex numbers $\lambda_{1}, \ldots, \lambda_{n}$ with $\lambda_{1}+\cdots+\lambda_{n}=0$ and every set $U_{1}, \ldots, U_{n} \in U_{\alpha}$ 
we have

$$
\sum_{i, j=1}^{n} \lambda_{i} \bar{\lambda}_{j} c\left(U_{i}, U_{j}\right) \geq 0 .
$$

Using (1.1), one may construct a Hilbert space $H(\alpha)$ as follows. Letting $\mathbb{C}_{0} U_{\alpha}$ denote the complex vector space of all finitely nonzero functions $f: U_{\alpha} \rightarrow \mathbb{C}$ satisfying

$$
\sum_{x} f(x)=0
$$

we define a sesquilinear form on $\mathbb{C}_{0} U_{\alpha}$ by

$$
\langle f, g\rangle=\sum_{x, y} f(x) \overline{g(y)} c(x, y) .
$$

$\langle\cdot, \cdot\rangle$ is positive semidefinite by $(1.1)$, and its kernel

$$
N=\left\{f \in \mathbb{C}_{0} U_{\alpha}:\langle f, f\rangle=0\right\}
$$

is a linear subspace of $\mathbb{C}_{0} U_{\alpha} \cdot\langle\cdot, \cdot\rangle$ induces an inner product on the quotient $\mathbb{C}_{0} U_{\alpha} / N$, and the completion of the latter is a Hilbert space $H(\alpha)$ which is necessarily separable (cf. [1], Proposition 5.2).

In case $\mathscr{U}_{\alpha} \neq \varnothing, d_{*}(\alpha)$ is defined as the dimension of $H(\alpha)$. Thus, $d_{*}(\alpha)$ belongs to $\{1,2, \ldots, \infty\}$ where the symbol $\infty$ stands for the cardinal $\aleph_{0}$. If $\mathscr{U}_{\alpha} \neq \varnothing$, it will be arithmetically convenient to define $d_{*}(\alpha)=c$, the cardinality of the continuum. The set $\{1,2, \ldots, \infty, c\}$ of values of $d_{*}$ is an abelian semigroup under addition, where the usual addition in $\{1,2, \ldots, \infty\}$ is extended to the set obtained from it by adjoining $c$ according to the rules

$$
x+c=c+x=c, \quad c+c=c,
$$

$x=1,2, \ldots, \infty$. It was shown in [1] that $d_{*}(\alpha)$ is an invariant for outer conjugacy of $E_{0}$-semigroups $\alpha$, and that if $\alpha$ and $\beta$ have the same index in the sense of Powers and Robinson [4] then $d_{*}(\alpha)=d_{*}(\beta)$. Moreover, if $\alpha$ is the CAR flow of rank $n$ then $d_{*}(\alpha)=n$ ([1], Corollary 2 of Proposition 5.3).

If $\alpha$ (resp. $\beta$ ) is an $E_{0}$-semigroup acting on $B(H)($ resp. $B(K))$, then there is a unique $E_{0}$-semigroup $\alpha \otimes \beta$ acting on $B(H \otimes K)$ such that

$$
(\alpha \otimes \beta)_{t}(A \otimes B)=\alpha_{t}(A) \otimes \beta_{t}(B),
$$

for all $A \in B(H), B \in B(K)$. It follows from the above remarks that $d_{*}$ obeys the logarithmic additivity property

$$
d_{*}(\alpha \otimes \beta)=d_{*}(\alpha)+d_{*}(\beta)
$$


whenever $\alpha$ and $\beta$ are outer conjugate to CAR flows. The purpose of this paper is to establish (1.2) in general. Equivalently, if $\mathscr{U}_{\alpha \otimes \beta}=\varnothing$ then either $\mathscr{U}_{\alpha}=\varnothing$ or $\mathscr{U}_{\beta}=\varnothing$; and if $\mathscr{U}_{\alpha \otimes \beta} \neq \varnothing$, then both $\mathscr{U}_{\alpha}$ and $\mathscr{U}_{\beta}$ are nonvoid and

$$
H(\alpha \otimes \beta) \cong H(\alpha) \oplus H(\beta) .
$$

The first of these two assertions is clear from the fact that if both $\mathscr{U}_{\alpha}$ and $\mathscr{U}_{\beta}$ are nonvoid and we choose $U \in \mathscr{U}_{\alpha}$ and $V \in \mathscr{U}_{\beta}$, then the semigroup $(U \otimes V)_{t}=U_{t} \otimes V_{t}$ belongs to $\mathscr{U}_{\alpha \otimes \beta}$ and hence $\mathscr{U}_{\alpha \otimes \beta} \neq \varnothing$. The second assertion (including (1.3)) is a consequence of Theorem 4.4 below. In particular, we show that every semigroup in $\mathscr{U}_{\alpha \otimes \beta}$ decomposes into a tensor product $U \otimes V$ where $U \in \mathscr{U}_{\alpha}$ and $V \in \mathscr{U}_{\beta}$.

We remark that while the above definition of $\mathscr{U}_{\alpha}$ (and therefore $H(\alpha)$ ) appears to differ from the definition of $\mathscr{U}_{\alpha}$ given in [1], it is actually the same. The proof of that amounts to showing that if $\left\{U_{t}: t>0\right\}$ is a weakly measurable family of bounded operators on $H$ satisfying $U_{s} U_{t}=U_{s+t}$ for $s, t>0$ and

$$
\alpha_{t}(A) U_{t}=U_{t} A, \quad A \in B(H), t>0,
$$

then $\left\{U_{t}: t>0\right\}$ is strongly continuous and $U_{t}$ tends strongly to 1 as $t \rightarrow 0+$. To see this, note that by ([1], Theorem 4.1) there is a real constant $a$ such that

$$
U_{t}^{*} U_{t}=e^{a t} 1, \quad t>0,
$$

and hence $V_{t}=e^{-1 / 2 a t} U_{t}$ is a measurable semigroup of isometries. The assertion now follows from ([1], Proposition 2.5(ii)).

2. Multipliers of $(0, \infty)$. By a multiplier of $(0, \infty)$ we mean a Borelmeasurable function $m:(0, \infty) \times(0, \infty) \rightarrow\{|z|=1\}$ satisfying

$$
m(x, y+z) m(y, z)=m(x+y, z) m(x, y), \quad x, y>0 .
$$

The purpose of this section is to establish that every multiplier $m$ of $(0, \infty)$ is trivial in the sense that there is a measurable function $f:(0, \infty) \rightarrow\{|z|=1\}$ satisfying

$$
m(x, y)=\frac{f(x+y)}{f(x) f(y)}, \quad x, y>0 .
$$

While this is analogous to a well-known fact about multipliers of the additive group $\mathbb{R}([5]$, Theorem 10.38$)$, we have been unable to find the result we need in the literature.

We will deduce (2.2) from the following representation theorem. In the proof, we use a familiar theorem which asserts that every weakly 
continuous one-parameter group $\gamma=\left\{\gamma_{t}: t \in \mathbb{R}\right\}$ of $*$-automorphisms of $B(H)$ is implemented by a strongly continuous one-parameter unitary group $U$ :

$$
\gamma_{t}(A)=U_{t} A U_{t}^{*}, \quad t \in \mathbb{R}, A \in B(H)
$$

(for example, see [5] p. 141). Of course, the proof of that makes essential use of the fact that $\mathbb{R}$ has no nontrivial multipliers.

Proposition 2.3. Let $\alpha=\left\{\alpha_{t}: t \geq 0\right\}$ be a $*$-semigroup acting on $B(H)$ such that each $\alpha_{t}$ leaves the set of compact operators invariant. Then there is a strongly continuous semigroup $\left\{V_{t}: t \geq 0\right\}$ of isometries in $B(H)$ such that

$$
\alpha_{t}(A)=V_{t} A V_{t}^{*}, \quad t \geq 0, A \in B(H) .
$$

Proof. For every $t>0$, consider the linear space of operators

$$
E_{t}=\left\{T \in B(H): \alpha_{t}(A) T=T A, A \in B(H)\right\} .
$$

$E_{t} \neq\{0\}$ and is a Hilbert space relative to the inner product $[\cdot, \cdot]$ defined on it by

$$
[S, T] 1=T^{*} S, \quad S, T \in E_{t} .
$$

Moreover, for each $s, t>0$ there is a natural unitary operator which maps $E_{s+t}$ onto $E_{s} \otimes E_{t}$ (for details, see $\S 2$ of [1]). So if $d(t)$ is the dimension of $E_{t}$, then $d$ satisfies the functional equation

$$
d(s+t)=d(s) d(t), \quad s, t>0 .
$$

The only solutions of (2.4) taking values in $\{1,2, \ldots, \infty\}$ are $d \equiv 1$ and $d \equiv \infty$. Notice that the case $d \equiv \infty$ cannot occur. For if $E_{t}$ is infinite dimensional and we choose an orthonormal basis $V_{1}, V_{2}, \ldots$ for $E_{t}$, then by ([1], Proposition 2.1) the $V_{n}$ 's are isometries having mutually orthogonal ranges which satisfy

$$
\alpha_{t}(A)=\sum_{n=1}^{\infty} V_{n} A V_{n}^{*}, \quad A \in B(H),
$$

and this contradicts the hypothesis that $\alpha_{t}$ should map compact operators to compact operators.

In particular, we must have $d(1)=1$. This means that $E_{1}=\mathbb{C} \cdot V$ where $V$ is an isometry which satisfies

$$
\alpha_{1}(A)=V A V^{*}, \quad A \in B(H) .
$$


Let $U$ be a minimal unitary extension of $V$. This is to say that $U$ is a unitary operator on a Hilbert space $\tilde{H}$ containing $H$ which satisfies

(i) $\frac{\left.U\right|_{H}=V}{\bigcup_{n \in \mathbb{Z}} U^{n} H}=\tilde{H}$.

Let $P \in B(\tilde{H})$ be the projection onto $H$. The map $A \in K(H) \mapsto$ $A P \in K(\tilde{H})$ is a *-monomorphism which identifies the compact operators on $H$ with the corner

$$
K_{0}=P K(\tilde{H}) P
$$

of the compact operators on $\tilde{H}$. Thus we may think of $\left\{\alpha_{t}: t \geq 0\right\}$ as a semigroup of $*$-endomorphisms of $K_{0} \subseteq K(\tilde{H})$ satisfying

$$
\lim _{t \rightarrow 0}\left\|\alpha_{t}(A)-A\right\|=0
$$

for every $A \in K_{0}$. Moreover, $\alpha_{1}(A)=U A U^{*}$ for $A \in K_{0}$.

Notice that there is a natural way to extend $\left\{\alpha_{t}\right\}$ to a semigroup $\left\{\beta_{t}\right\}$ of *-endomorphisms of the $C^{*}$-algebra $K(\tilde{H})$ of compact operators on $\tilde{H}$. To see this, let

$$
K_{n}=U^{n} K_{0} U^{* n}, \quad n \in \mathbb{Z} .
$$

We have $K_{n+1} \subset K_{n}$, and as $n$ decreases to $-\infty$ the $C^{*}$-algebras $K_{n}$ increase to a dense $*$-subalgebra of $K(\tilde{H})$. For each $n \leq 0$ we can define a semigroup $\left\{\beta_{t}: t \geq 0\right\}$ of $*$-endomorphisms of $K_{n}$ by

$$
\beta_{t}(A)=U^{n} \alpha_{t}\left(U^{-n} A U^{n}\right) U^{-n},
$$

$A \in K_{n}, t \geq 0 . \quad\left\{\beta_{t}\right\}$ is clearly conjugate to $\left\{\alpha_{t}\right\}$. Moreover, the restriction of $\beta_{t}$ to $K_{0}$ is $\alpha_{t}$ since for $A \in K_{0}$ we have

$$
\begin{aligned}
\beta_{t}(A) & =U^{n} \alpha_{t}\left(\alpha_{-n}(A)\right) U^{-n} \\
& =U^{n} \alpha_{t-n}(A) U^{-n}=U^{n} \alpha_{-n}\left(\alpha_{t}(A)\right) U^{-n} \\
& =U^{n} U^{-n}\left(\alpha_{t}(A)\right) U^{n} U^{-n}=\alpha_{t}(A) .
\end{aligned}
$$

Similarly, one checks that the defintion of $\beta_{t}$ on $K_{n-1}$ agrees with the definition of $\beta_{t}$ on the smaller algebra $K_{n}$ for every $n \leq 0$. Hence $\beta_{t}$ is well-defined on the dense $*$-subalgebra $K_{0} \cup K_{-1} \cup K_{-2} \cup \cdots$ of $K(\tilde{H})$. So $\left\{\beta_{t}: t \geq 0\right\}$ extends uniquely to a semigroup of $*$-endomorphisms of $K(\tilde{H})$ satisfying

(i) $\lim _{t \rightarrow 0}\left\|\beta_{t}(A)-A\right\|=0$,

(ii) $\left.\beta_{t}\right|_{K_{0}}=\alpha_{t}$, and

(iii) $\beta_{t}\left(U B U^{-1}\right)=U \beta_{t}(B) U^{-1}, \quad B \in K(\tilde{H}), t \geq 0$. 
We claim that $\beta_{1}(K(\tilde{H}))=K(\tilde{H})$. Indeed, $\beta_{1}(K(\tilde{H}))$ is a $C^{*}$-subalgebra of $K(\tilde{H})$ which is invariant under the automorphism $B \mapsto$ $U^{-1} B U$ and which contains

$$
\beta_{1}\left(K_{0}\right)=\alpha_{1}\left(K_{0}\right)=V K_{0} V^{*}=U K_{0} U^{-1}=K_{1} .
$$

Hence $\beta_{1}(K(\tilde{H}))$ contains $K_{1} \cup K_{0} \cup K_{-1} \cup \cdots$, and the claim follows since the latter is dense in $K(\tilde{H})$.

By the semigroup property we conclude that $\beta_{t}(K(\tilde{H}))=K(\tilde{H})$ for every $t \geq 0$ and this implies that every $\beta_{t}$ is a $*$-automorphism of $K(\tilde{H})$. Extending $\beta_{t}$ to negative values of $t$ by $\beta_{t}=\beta_{|t|}$, we obtain a $C^{*}$-dynamical system $(K(\tilde{H}), \mathbb{R}, \beta)$ which extends naturally to a $W^{*}$ dynamical system $(B(\tilde{H}), \mathbb{R}, \beta)$. By the preceding remarks there is a strongly continuous one-parameter unitary group $\left\{W_{t}: t \in \mathbb{R}\right\}$ acting on $\tilde{H}$ such that

$$
\beta_{t}(B)=W_{t} B W_{t}^{-1}, \quad t \geq 0, B \in B(H) .
$$

For $t \geq 0, \beta_{t}$ leaves the corner $K_{0}=P K(\tilde{H}) P$ invariant and hence $W_{t} P W_{t}^{-1} \leq P$. It follows that the subspace $H=P \tilde{H}$ is invariant under $\left\{W_{t}: t \geq 0\right\}$, and we obtain the desired semigroup of isometries $\left\{V_{t}: t \geq 0\right\}$ by setting $V_{t}=\left.W_{t}\right|_{H}$.

Corollary. Let $m:(0, \infty) \times(0, \infty) \rightarrow\{|z|=1\}$ be a Borel-measurable function satisfying the multiplier equation

$$
m(x, y+z) m(y, z)=m(x+y, z) m(x, y), \quad x, y, z>0 .
$$

Then there is a measurable function $f:(0, \infty) \rightarrow\{|z|=1\}$ such that

$$
m(x, y)=\frac{f(x+y)}{f(x) f(y)}, \quad x, y>0
$$

Proof. For every $t>0$, define an operator $U_{t}$ on $L^{2}(0, \infty)$ by

$$
U_{t} f(x)= \begin{cases}m(t, x-t) f(x-t), & x>t, \\ 0, & 0 \leq x \leq t .\end{cases}
$$

$\left\{U_{t}: t>0\right\}$ is a measurable family of isometries which, because of the multiplier equation for $m$, satisfies

$$
U_{s} U_{t}=m(s, t) U_{s+t}, \quad s, t>0 .
$$

Therefore, $\alpha_{t}(A)=U_{t} A U_{t}^{*}$ defines a semigroup $\left\{\alpha_{t}: t>0\right\}$ of *endomorphisms of $B\left(L^{2}(0, \infty)\right)$ such that

$$
t \in(0, \infty) \mapsto\left\langle\alpha_{t}(A) f, g\right\rangle
$$


is measurable for fixed $f, g \in L^{2}(0, \infty)$ and $A \in B\left(L^{2}(0, \infty)\right)$. By ([1], Proposition 2.5(i)) the functions (2.7) are continuous and $\alpha_{t}(A) \rightarrow A$ weakly as $t \rightarrow 0+$ for every bounded operator $A$. So if we define $\alpha_{0}(A)=A$ for all $A$, then $\left\{\alpha_{t}: t \geq 0\right\}$ satisfies the hypothesis of Proposition 2.3.

Hence there is a strongly continuous semigroup $\left\{V_{t}: t \geq 0\right\}$ of isometries on $L^{2}(0, \infty)$ such that $\alpha_{t}(A)=V_{t} A V_{t}^{*}$, i.e.,

$$
U_{t} A U_{t}^{*}=V_{t} A V_{t}^{*}, \quad A \in B\left(L^{2}(0, \infty)\right), t>0 .
$$

Fix $t>0$. (2.8) implies that $U_{t}^{*} V_{t}$ commutes with every bounded operator and hence there is a scalar $f(t)$ such tht $U_{t}^{*} V_{t}=f(t) 1 . f$ is measurable because of the measurability of $U$ and $V$. Taking $A=1$ in (2.8) we obtain $U_{t} U_{t}^{*}=V_{t} V_{t}^{*}$ and hence $V_{t}=U_{t} U_{t}^{*} V_{t}=f(t) U_{t}$ for every $t>0$. Thus, $|f(t)|=1$ and, for every $s, t>0$ we have

$$
\begin{aligned}
f(s+t) U_{s+t} & =V_{s+t}=V_{s} V_{t}=f(s) f(t) U_{s} U_{t} \\
& =f(s) f(t) m(s, t) U_{s+t} .
\end{aligned}
$$

The required formula follows by multiplying the latter equation on the left by $U_{s+t}^{*}$.

3. Compact morphisms of product systems. We begin by recalling the definition of a (continuous) product system. This is a measurable family of separable infinite dimensional Hilbert spaces

$$
p: E \rightarrow(0, \infty)
$$

which is endowed with a measurable associative multiplication

$$
x, y \in E \times E \mapsto x y \in E
$$

which acts like tensoring in the following sense. Letting $E_{t}=p^{-1}(t)$ be the Hilbert space over $t \in(0, \infty)$, we require that

(i) $E_{s+t}=\overline{\operatorname{span}} E_{s} E_{t}, \quad s, t>0$, and

(ii) for all $x, x^{\prime} \in E_{s}$ and $y, y^{\prime} \in E_{t}$, one has $\left\langle x y, x^{\prime} y^{\prime}\right\rangle=\left\langle x, x^{\prime}\right\rangle\left\langle y, y^{\prime}\right\rangle$.

In more detail, the symbol $E$ in (3.1) denotes a standard Borel space, $p$ denotes a measurable surjection such that each fiber $E_{t}=p^{-1}(t)$ is endowed with the structure of a complex Hilbert space, in such a way that there is a separable infinite dimensional Hilbert space $H_{0}$ and a Borel isomorphism

$$
\theta: E \rightarrow(0, \infty) \times H_{0}
$$


of $E$ onto the indicated trivial family which commutes the diagram

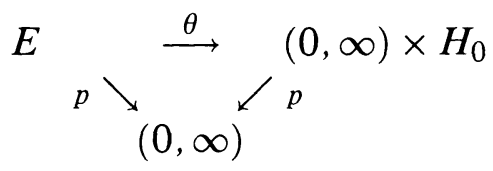

and is unitary on fiber spaces.

By a unit of $E$ we mean a measurable section

$$
t \in(0, \infty) \mapsto u_{t} \in E_{t}
$$

such that $u_{s+t}=u_{s} u_{t}$ for all $s, t>0$, and which is not the zero section. $\mathscr{U}_{E}$ will denote the set of units of $E$. Finally, a morphism of product systems is a Borel map of product systems $\theta: E \rightarrow F$ such that $\theta(x y)=\theta(x) \theta(y)$ for all $x, y \in E$ and such that the restriction $\theta_{t}=\left.\theta\right|_{E_{t}}$ of $\theta$ to each fiber $E_{t}$ is a bounded linear operator from $E_{t}$ to $F_{t}$ for every $t>0$. A morphism $\theta$ is called compact if each $\theta_{t}$ is a compact operator.

If one is given a pair of units $u \in \mathscr{U}_{E}$ and $v \in \mathscr{U}_{F}$, then one can define a morphism $\theta: E \rightarrow F$ as follows:

$$
\theta(x)=\left\langle x, u_{t}\right\rangle v_{t}, \quad x \in E_{t}, t>0 .
$$

$\theta$ is compact because each $\theta_{t}$ is of rank at most one. The purpose of this section is to establish the following result, which asserts that these are the only compact morphisms. This will allow us to identify the units of a tensor product $E \otimes F$ of product systems in Corollary 3.9.

Theorem 3.4. Let $E$ and $F$ be product systems and let $\theta: E \rightarrow F$ be a compact morphism such that $\theta_{t_{0}} \neq 0$ for some $t_{0}>0$. Then there exist units $u \in \mathscr{U}_{E}, v \in \mathscr{U}_{F}$ such that

$$
\theta_{t}(x)=\left\langle x, u_{t}\right\rangle v_{t}, \quad x \in E_{t}, t>0 .
$$

Proof. We first consider the case in which $F=E$ and each $\theta_{t}$ is a positive compact operator in $B\left(E_{t}\right), t>0$. We will show that there is a unit $u$ in $\mathscr{U}_{E}$ such that

$$
\theta_{t}(x)=\left\langle x, u_{t}\right\rangle u_{t}, \quad x \in E_{t}, t>0 .
$$

Note first that $\left\|\theta_{s+t}\right\|=\left\|\theta_{s}\right\| \cdot\left\|\theta_{t}\right\|$ for every $s, t>0$. Indeed, (3.2) implies that there is a unitary operator

$$
W_{s, t}: E_{s+t} \rightarrow E_{s} \otimes E_{t}
$$

which implements a unitary equivalence of the maps $\theta_{s+t} \in B\left(E_{s+t}\right)$ and $\theta_{s} \otimes \theta_{t} \in B\left(E_{s} \otimes E_{t}\right)$, from which the assertion is evident. The 
function $t \in(0, \infty) \mapsto\left\|\theta_{t}\right\|$ is clearly measurable and is nonzero at $t_{0}>0$. It follows from a simple argument (see the proof of Theorem 4.1 of [1]) that there is a real constant $a$ such that

$$
\left\|\theta_{t}\right\|=e^{a t}, \quad t>0 .
$$

So by replacing $\theta_{t}$ with $e^{-a t} \theta_{t}$ if necessary, we can assume that $\left\|\theta_{t}\right\|=$ 1 for every positive $t$.

For each $t>0$ let $e_{t}$ be the projection of $E_{t}$ onto the nonzero finite dimensional subspace

$$
\left\{\xi \in E_{t}: \theta_{t} \xi=\xi\right\} \text {. }
$$

Since the sequence $\theta_{t}, \theta_{t}^{2}, \theta_{t}^{3}, \ldots$ converges strongly to $e_{t}$ for every positive $t$, it follows that $t \mapsto e_{t}$ is a measurable family of operators.

We claim that each $e_{t}$ is one-dimensional. To see this, note that for every $s, t>0$ the unitary equivalence $\theta_{s+t} \cong \theta_{s} \otimes \theta_{t}$ implies that $\theta_{s+t}^{n} \cong \theta_{s}^{n} \otimes \theta_{t}^{n}$ for every $n \geq 1$, and hence $e_{s+t} \cong e_{s} \otimes e_{t}$. So the dimension $d(t)$ of $e_{t}$ satisfies the functional equation

$$
d(s+t)=d(s) d(t), \quad s, t>0 .
$$

The only solution of the latter, taking values in $\{1,2, \ldots\}$, is the function $d(t)=1, t>0$, and the claim is proved.

We claim next that there is a measurable section $t \in(0, \infty) \mapsto \xi_{t} \in$ $E_{t}$ of unit vectors such that $e_{t}\left(\xi_{t}\right)=\xi_{t}, t>0$. To prove this, we may assume by $(3.3)$ that $E$ is the trivial family $(0, \infty) \times H_{0}$ and that $e_{t}$ is a one-dimensional projection in $B\left(H_{0}\right)$ for every $t>0$. Choose an orthonormal basis $\zeta_{1}, \zeta_{2}, \ldots$ for $H_{0}$. For each $t>0$, define $n(t)$ to be the smallest positive integer $k$ such that $e_{t}\left(\zeta_{k}\right) \neq 0$. The function $n:(0, \infty) \rightarrow \mathbb{N}$ is measurable, and therefore

$$
\xi_{t}=\frac{e_{n(t)}\left(\zeta_{n(t)}\right)}{\left\|e_{n(t)}\left(\zeta_{n(t)}\right)\right\|}, \quad t>0
$$

defines a measurable section with the asserted properties.

We now show that $\xi_{t}$ has the form

$$
\xi_{t}=f(t) u_{t},
$$

where $u$ is a unit of $E$ and $f:(0, \infty) \rightarrow \mathbb{C}$ is a measurable function satisfying $|f(t)|=1$ for every $t>0$. To prove this, we claim first that $\xi_{s} \xi_{t}$ is proportional to $\xi_{s+t}$ for every $s, t>0$. Indeed,

$$
\theta_{s+t}\left(\xi_{s} \xi_{t}\right)=\theta_{s}\left(\xi_{s}\right) \theta_{t}\left(\xi_{t}\right)=\xi_{s} \xi_{t}
$$


so that $\xi_{s} \xi_{t}$ is a unit vector in the range of $e_{s+t}$. The claim follows from the fact that $e_{s+t}$ is one-dimensional.

Thus, there is a unique function $m:(0, \infty) \times(0, \infty) \rightarrow\{|z|=1\}$ such that

$$
\xi_{s} \xi_{t}=m(s, t) \xi_{s+t}
$$

$m$ is clearly measurable. Note that $m$ must satisfy the multiplier equation

$$
m(r, s+t) m(s, t)=m(r+s, t) m(r, s), \quad r, s, t>0 .
$$

Indeed, fixing $r, s, t>0$ and using associativity of the multiplication in $E$ we have

$$
m(r, s+t) m(s, t) \xi_{r} \xi_{s} \xi_{t}=m(r, s+t) \xi_{r} \xi_{s+t}=\xi_{r+s+t},
$$

whereas

$$
m(r+s, t) m(r, s) \xi_{r} \xi_{s} \xi_{t}=m(r+s, t) \xi_{r+s} \xi_{t}=\xi_{r+s+t},
$$

and (3.6) follows.

By Proposition 2.3 there is a measurable function $f:(0, \infty) \rightarrow$ $\{|z|=1\}$ such that

$$
m(s, t)=f(s+t) / f(s) f(t), \quad s, t>0 .
$$

If we define $u_{t}=f(t) \xi_{t}$, then $\left\|u_{t}\right\|=1$ for all $t>0$ and (3.5) implies that $u$ is a unit.

It follows that $\theta_{t}$ can be decomposed as an orthogonal sum of operators

$$
\theta_{t}=e_{t}+\rho_{t}
$$

where $e_{t}$ is the one-dimensional projection $e_{t}(x)=\left\langle x, u_{t}\right\rangle u_{t}$ and where $\left\{\rho_{t}: t>0\right\}$ is a measurable family of positive compact operators satisfying $\left\|\rho_{t}\right\|<1$ and $\rho_{t} e_{t}=e_{t} \rho_{t}=0, t>0$.

It remains to show that each $\rho_{t}$ is zero. Fix $s, t>0$. We have seen that $\theta_{s+t}$ is unitarily equivalent to $\theta_{s} \otimes \theta_{t}$, and hence $e_{s+t}+\rho_{s+t}$ is unitarily equivalent to the direct sum

$$
\left(e_{s} \otimes e_{t}\right) \oplus\left(e_{s} \otimes \rho_{t}\right) \oplus\left(\rho_{s} \otimes e_{t}\right) \oplus\left(\rho_{s} \otimes \rho_{t}\right) .
$$

Because $u_{s+t}=u_{s} u_{t}, e_{s+t}$ is identified with $e_{s} \otimes e_{t}$ in the above unitary equivalence, and hence $\rho_{s+t}$ is unitarily equivalent to the direct sum

$$
\left(e_{s} \otimes \rho_{t}\right) \oplus\left(\rho_{s} \otimes e_{t}\right) \oplus\left(\rho_{s} \otimes \rho_{t}\right) .
$$


Taking $s=t$ and noting that $\left\|\rho_{t}\right\|<1$ for all $t>0$, we conclude that if $\rho_{2 t} \neq 0$, then necessarily $\rho_{t} \neq 0$ and $\left\|\rho_{2 t}\right\|=\left\|\rho_{t}\right\|$. Moreover, if $d(s)$ is the dimension of the eigenspace

$$
\left\{\xi \in E_{s}: \rho_{s}(\xi)=\left\|\rho_{s}\right\| \xi\right\},
$$

then we may also conclude that $d(2 t)=2 d(t)$ whenever $\rho_{2 t} \neq 0$.

Now suppose there is a $t_{0}>0$ such that $\rho_{t_{0}} \neq 0$. The preceding paragraph implies that

$$
d\left(t_{0}\right)=2 d\left(t_{0} / 2\right)=4 d\left(t_{0} / 4\right)=\cdots=2^{n} d\left(t_{0} / 2^{n}\right)
$$

for every $n \geq 1$. Since $d\left(t_{0} / 2^{n}\right)$ is a positive integer we conclude that $2^{n}$ divides $d\left(t_{0}\right)$ for every $n \geq 1$, which is absurd.

This completes the proof in the case where $F=E$ and each $\theta_{t}$ is a positive operator. Now suppose more generally that $\theta: E \rightarrow F$ is an arbitrary morphism such that $\theta_{t}: E_{t} \rightarrow F_{t}$ is compact for every $t>0$. The adjoint $\theta^{*}=\left\{\theta_{t}^{*}: t>0\right\}$ defines a measurable family of compact operators from $F$ to $E$. We claim that $\theta^{*}$ is a morphism, i.e.,

$$
\theta_{s+t}^{*}(x y)=\theta_{s}^{*}(x) \theta_{t}^{*}(y), \quad x \in F_{s}, y \in F_{t},
$$

for every $s, t>0$. To see this, fix $s$ and $t$ and choose $x \in F_{s}, y \in F_{t}$, $x^{\prime} \in E_{s}, y^{\prime} \in E_{t}$. We have

$$
\begin{aligned}
\left\langle\theta_{s+t}^{*}(x y), x^{\prime} y^{\prime}\right\rangle & =\left\langle x y, \theta_{s+t}\left(x^{\prime} y^{\prime}\right)\right\rangle \\
& =\left\langle x y, \theta_{s}\left(x^{\prime}\right) \theta_{t}\left(y^{\prime}\right)\right\rangle=\left\langle x, \theta_{s}\left(x^{\prime}\right)\right\rangle\left\langle y, \theta_{t}\left(y^{\prime}\right)\right\rangle \\
& =\left\langle\theta_{s}^{*}(x), x^{\prime}\right\rangle\left\langle\theta_{t}^{*}(y), y^{\prime}\right\rangle=\left\langle\theta_{s}^{*}(x) \theta_{t}^{*}(y), x^{\prime} y^{\prime}\right\rangle .
\end{aligned}
$$

(3.8) follows because $E_{s} E_{t}$ spans $E_{s+t}$.

Therefore $\omega_{t}=\theta_{t}^{*} \theta_{t}, t>0$, defines a morphism of $E$ consisting of positive compact operators, not all of which are zero. By the above argument, there is a unit $u \in \mathscr{U}_{E}$ satisfying $\left\|u_{t}\right\|=1$ and a real number $a$ such that

$$
\omega_{t}(x)=e^{a t}\left\langle x, u_{t}\right\rangle u_{t}, \quad x \in E_{t}, t>0 .
$$

It follows that the initial space of $\theta_{t}$ is the one-dimensional space spanned by $u_{t}, t>0$. Put $v_{t}=\theta_{t}\left(u_{t}\right) . v$ is a unit of $F$ because $u$ is a unit of $E$ and $\theta$ is a morphism. It follows that for every $x \in E_{1}$ we have

$$
\theta_{t}(x)=\theta_{t}\left(\left\langle x, u_{t}\right\rangle u_{t}\right)=\left\langle x, u_{t}\right\rangle v_{t}
$$

as required.

Now let $E, F$ be two product systems and let $E \otimes F=\left\{E_{t} \otimes F_{t}: t>0\right\}$ 
be the tensor product of product systems (cf. [1], §3). The multiplication in $E \otimes F$ is defined uniquely by requiring

$$
(x \otimes y)(u \otimes v)=(x u) \otimes(y v),
$$

for $x \in E_{s}, y \in F_{s}, u \in E_{t}, v \in F_{t}, s, t>0$. If $u \in \mathscr{U}_{E}$ and $v \in \mathscr{U}_{F}$ are units then one can define a unit $u \otimes v$ of $E \otimes F$ by $(u \otimes v)_{t}=u_{t} \otimes v_{t}$, $t>0$.

Corollary 3.9. Let $E$ and $F$ be product systems. Then every unit of $E \otimes F$ decomposes as a tensor product $u \otimes v$ where $u$ and $v$ are units of $E$ and $F$ respectively.

Proof. Let $\bar{F}$ be the conjugate of the product system $F$, i.e., $\bar{F}$ consists of the same family of Hilbert spaces $p: F \rightarrow(0, \infty)$ except that scalar multiplication in the fibers of $\bar{F}$ is conjugated: thus for $\lambda \in \mathbb{C}$ and $x \in \bar{F}_{t}, \lambda \cdot x$ means $\bar{\lambda} x$ rather than $\lambda x$. The multiplications in $\bar{F}$ and $F$ are the same. The identity map of $F$ can be considered a Borel isomorphism of $F$ on $\bar{F}$ which we denote by $x \mapsto \bar{x}$. This map preserves multiplication and is anti-unitary on the fiber spaces. The inner product in $\bar{F}_{t}$ is given by $\langle\bar{x}, \bar{y}\rangle=\langle y, x\rangle, x, y \in F_{t}$.

Now let $w$ be a unit of $E \otimes F$. For each $t>0$, the bounded bilinear map

$$
x, y \in E_{t} \times F_{t} \mapsto\left\langle x \otimes y, w_{t}\right\rangle
$$

can be viewed as a sesquilinear map on $E_{t} \times \bar{F}_{t}$. Thus there is a unique bounded linear operator $\theta_{t}: E_{t} \rightarrow \bar{F}_{t}$ such that

$$
\left\langle\theta_{t}(x), \bar{y}\right\rangle=\left\langle x \otimes y, w_{t}\right\rangle, \quad x \in E_{t}, y \in F_{t} .
$$

Notice that $\theta: E \rightarrow \bar{F}$ is a morphism. Indeed $\theta$ is clearly a measurable family of bounded linear operators and it is multiplicative because if $x \in E_{s}, x^{\prime} \in E_{t}$ then for every vector in $F_{s+t}$ of the form $y y^{\prime}$ with $y \in F_{s}$ and $y^{\prime} \in F_{t}$ we have

$$
\begin{aligned}
\left\langle\theta_{s+t}\left(x x^{\prime}\right), \overline{y y^{\prime}}\right\rangle & =\left\langle x x^{\prime} \otimes y y^{\prime}, w_{s+t}\right\rangle \\
& =\left\langle(x \otimes y)\left(x^{\prime} \otimes y^{\prime}\right), w_{s} w_{t}\right\rangle \\
& =\left\langle x \otimes y, w_{s}\right\rangle\left\langle x^{\prime} \otimes y^{\prime}, w_{t}\right\rangle=\left\langle\theta_{s}(x), \bar{y}\right\rangle\left\langle\theta_{t}\left(x^{\prime}\right), \overline{y^{\prime}}\right\rangle \\
& =\left\langle\theta_{s}(x) \theta_{t}\left(x^{\prime}\right), \bar{y} \overline{y^{\prime}}\right\rangle=\left\langle\theta_{s}(x) \theta_{t}\left(x^{\prime}\right), \overline{y y^{\prime}}\right\rangle .
\end{aligned}
$$

The assertion follows because $F_{s+t}$ is spanned by $F_{s} F_{t}$. 
We claim that each $\theta_{t}$ is a Hilbert-Schmidt operator. Indeed, if $\xi_{1}$, $\xi_{2}, \ldots$ (resp. $\left.\eta_{1}, \eta_{2}, \ldots\right)$ is an orthonormal basis for $E_{t}$ (resp. $F_{t}$ ) then

$$
\begin{aligned}
\sum_{m}\left\|\theta_{t}\left(\xi_{m}\right)\right\|^{2} & =\sum_{m, n}\left|\left\langle\theta_{t}\left(\xi_{m}\right), \bar{\eta}_{n}\right\rangle\right|^{2} \\
& =\sum_{m, n}\left|\left\langle\xi_{m} \otimes \eta_{n}, w_{t}\right\rangle\right|^{2}=\left\|w_{t}\right\|^{2}<\infty,
\end{aligned}
$$

because $\left\{\xi_{m} \otimes \eta_{n}: m, n \geq 1\right\}$ is an orthonormal basis for $E_{t} \otimes F_{t}$.

Thus, $\theta$ is a compact morphism. By Theorem 3.4 and the fact that every unit $v$ of $\bar{F}$ has the form $v_{t}=\bar{w}_{t}$ for some unit of $w$ of $F$, we conclude that there are units $u \in \mathscr{U}_{E}$ and $v \in \mathscr{U}_{F}$ such that

$$
\theta_{t}(x)=\left\langle x, u_{t}\right\rangle \overline{v_{t}}, \quad x \in E_{t}, t>0 .
$$

Substitution of the latter in (3.10) gives

$$
\left\langle\left\langle x, u_{t}\right\rangle \overline{v_{t}}, \bar{y}\right\rangle=\left\langle x \otimes y, w_{t}\right\rangle, \quad x \in E_{t}, y \in F_{t} .
$$

The left side can be written

$$
\left\langle x, u_{t}\right\rangle\left\langle\overline{v_{t}}, \bar{y}\right\rangle=\left\langle x, u_{t}\right\rangle\left\langle y, v_{t}\right\rangle=\left\langle x \otimes y, u_{t} \otimes v_{t}\right\rangle .
$$

It follows that $w_{t}=u_{t} \otimes v_{t}$, as asserted.

REMARK. Referring back to the context of the introduction, let $\left\{\alpha_{t}: t \geq 0\right\}$ and $\left\{\beta_{t}: t \geq 0\right\}$ be $E_{0}$-semigroups acting on $B(H)$ and $B(K)$ respectively, and let $\left\{W_{t}: t \geq 0\right\}$ be a semigroup of isometries in $B(H \otimes K)$ such that

$$
(\alpha \otimes \beta)_{t}(A) W_{t}=W_{t} A, \quad A \in B(H \otimes K), t \geq 0 .
$$

Then there are semigroups of isometries $U$ in $\mathscr{U}_{\alpha}$ and $V$ in $\mathscr{U}_{\beta}$ such that $W_{t}=U_{t} \otimes V_{t}$ for every $t \geq 0$. This follows from Corollary 3.9 together with the basic results on the relation between an $E_{0}$-semigroup $\gamma=\left\{\gamma_{t}: t \geq 0\right\}$ and its associated product system ([1], $\left.\S 2\right)$.

4. Dimension and index. We now apply the results of $\S 3$ to prove an addition theorem for the dimension of product systems and the index of $E_{0}$-semigroups.

Let $E$ be a product system and let $\mathscr{U}_{E}$ be its set of units. Theorem 4.1 of [1] asserts that for every pair of units $u, v \in \mathscr{U}_{E}$ there is a complex number $c(u, v)$ such that

$$
\left\langle u_{t}, v_{t}\right\rangle=e^{t c(u, v)}, \quad t>0 .
$$

$c: \mathscr{U}_{E} \times \mathscr{U}_{E} \rightarrow \mathbb{C}$ is called the covariance function of $E$ and it is selfadjoint and conditionally positive definite. As in the introduction, one 
can use $c$ to construct a (necessarily separable) Hilbert space $H_{E}$. The dimension of $E$ is defined as follows

$$
\operatorname{dim} E= \begin{cases}\operatorname{dim} H_{E}, & \text { if } \mathscr{U}_{E} \neq \varnothing, \\ c, & \text { if } \mathscr{U}_{E}=\varnothing .\end{cases}
$$

Notice that (4.1) differs slightly from the definition given in $\S 5$ of [1]; in the former we define $\operatorname{dim} E$ to be 0 in the exceptional case where $\mathscr{U}_{E}=\varnothing$. The present definition leads to somewhat more attractive algebraic formulas.

Before presenting the main results we prove a simple lemma about abstract covariance functions which will facilitate the computation of $\operatorname{dim} E$. By an (abstract) covariance function we mean a pair $(X, c)$ consisting of a nonvoid set $X$ and a function $c: X \times X \rightarrow \mathbb{C}$ satisfying

(i) $\overline{c(x, y)}=c(y, x)$, and

(ii) $\sum_{i, j=1}^{n} \lambda_{i} \overline{\lambda_{j}} c\left(x_{i}, x_{j}\right) \geq 0$

for all $\lambda_{1}, \ldots, \lambda_{n} \in \mathbb{C}$ satisfying $\lambda_{1}+\cdots+\lambda_{n}=0$, all $x_{1}, \ldots, x_{n} \in$ $X$, and all $n=1,2, \ldots$. Starting with the vector space $\mathbb{C}_{0} X$ of all finitely nonzero functions $f: X \rightarrow \mathbb{C}$ satisfying $\sum_{x} f(x)=0$, we can construct a Hilbert space $H(X, c)$ by the same method sketched in the introduction (for more detail, in $\S 5$ of [1]).

We define the direct sum of two covariance functions $(X, a)$ and $(Y, b)$ to be the covariance function $(X \times Y, c)$ where $c:(X \times Y) \times$ $(X \times Y) \rightarrow \mathbb{C}$ is defined by

$$
c\left((x, y),\left(x^{\prime}, y^{\prime}\right)\right)=a\left(x, x^{\prime}\right)+b\left(y, y^{\prime}\right) .
$$

LEMMA 4.3. Let $(X, a),(Y, b)$ be covariance functions.

(i) If there exists a surjective function $\theta: X \rightarrow Y$ such that $b(\theta x, \theta y)$ $=a(x, y)$ for all $x, y \in X$, then

$$
\operatorname{dim} H(X, a)=\operatorname{dim} H(Y, b) .
$$

(ii) If $(X \times Y, c)$ is the direct sum of $(X, a)$ and $(Y, b)$, then

$$
\operatorname{dim} H(X \times Y, c)=\operatorname{dim} H(X, a)+\operatorname{dim} H(Y, b) .
$$

Proof. To prove (i), we will construct a unitary operator from $H(X, a)$ to $H(Y, b)$. Define sesquilinear forms $\langle\cdot, \cdot\rangle$ and $\langle\cdot, \cdot\rangle^{\prime}$ on $\mathbb{C}_{0} X$ and $\mathbb{C}_{0} Y$ respectively by

$$
\begin{aligned}
\langle f, g\rangle & =\sum_{x, y \in X} f(x) \overline{g(y)} a(x, y), \\
\langle h, k\rangle^{\prime} & =\sum_{u, v \in Y} h(u) \overline{k(v)} b(u, v) .
\end{aligned}
$$


Both are positive semidefinite, and

$$
\begin{aligned}
N & =\left\{f \in \mathbb{C}_{0} X:\langle f, f\rangle=0\right\}, \\
N^{\prime} & =\left\{g \in \mathbb{C}_{0} Y:\langle g, g\rangle^{\prime}=0\right\}
\end{aligned}
$$

are subspaces of $\mathbb{C}_{0} X$ and $\mathbb{C}_{0} Y$ respectively such that $\langle\cdot, \cdot\rangle$ and $\langle\cdot, \cdot\rangle^{\prime}$ induce inner products on $\mathbb{C}_{0} X / N$ and $\mathbb{C}_{0} Y / N^{\prime} . H(X, a)$ and $H(Y, b)$ are the respective completions of these inner product spaces.

We can define a linear transformation $W_{0}: \mathbb{C}_{0} X \rightarrow \mathbb{C}_{0} Y$ by

$$
W_{0}(f)=\sum_{x \in X} f(x) \delta_{\theta x},
$$

$\delta_{y}$ denoting the function with value 1 at $y \in Y$ and 0 elsewhere. Noting that

$$
\sum_{y \in Y} W_{0}(f)(y)=\sum_{y \in Y}\left(\sum_{\theta x=y} f(x)\right)=\sum_{x} f(x)=0
$$

we see that $W_{0}$ does not indeed map $\mathbb{C}_{0} X$ into $\mathbb{C}_{0} Y$. We claim that $W_{0}$ is surjective. Indeed, if $g$ is any nonzero element of $\mathbb{C}_{0} Y$ and $\left\{y_{1}, \ldots, y_{n}\right\}$ is the set of points where $g \neq 0$, then we may find $x_{1}, \ldots, x_{n}$ in $X$ such that $\theta x_{i}=y_{i}, 1 \leq i \leq n$. Putting

$$
f=\sum_{i=1}^{n} g\left(y_{i}\right) \delta_{x_{i}}
$$

we have $f \in \mathbb{C}_{0} X$ and $W_{0}(f)=g$.

Notice next that $\left\langle W_{0}(f), W_{0}(g)\right\rangle^{\prime}=\langle f, g\rangle$ for all $f, g \in \mathbb{C}_{0} X$. For we can write

$$
\begin{aligned}
\left\langle W_{0}(f), W_{0}(g)\right\rangle & =\sum_{y, y^{\prime} \in Y}\left(\sum_{\begin{array}{c}
\theta x=y \\
\theta x^{\prime}=y^{\prime}
\end{array}} f(x) \overline{g\left(x^{\prime}\right)} b\left(\theta x, \theta x^{\prime}\right)\right) \\
& =\sum_{y, y^{\prime}}\left(\sum_{\begin{array}{c}
\theta x=y \\
\theta x^{\prime}=y^{\prime}
\end{array}} f(x) \overline{g\left(x^{\prime}\right)} a\left(x, x^{\prime}\right)\right) \\
& =\sum_{x, x^{\prime}} f(x) \overline{g\left(x^{\prime}\right)} a\left(x, x^{\prime}\right)=\langle f, g\rangle,
\end{aligned}
$$

as asserted. It follows that $W_{0}(N) \subseteq N^{\prime}$ and that $W_{0}$ induces a surjective isometry

$$
W: \mathbb{C}_{0} X / N \rightarrow \mathbb{C}_{0} Y / N^{\prime}
$$


The closure of $W$ is the required unitary operator from $H(x, a)$ to $H(Y, b)$.

To prove (ii), we exhibit a unitary operator $V$ from $H(X \times Y, c)$ to $H(X, a) \oplus H(Y, b)$. First, we define a linear transformation $V_{0}$ from $\mathbb{C}_{0}(X \times Y)$ into the direct sum of vector spaces $\mathbb{C}_{0} X+\mathbb{C}_{0} Y$ by $V_{0}(f)=$ $\left(f_{1}, f_{2}\right)$ where

$$
f_{1}(x)=\sum_{y \in Y} f(x, y), \quad f_{2}(y)=\sum_{x \in X} f(x, y) .
$$

Note that $V_{0}$ is surjective. For if $f \in \mathbb{C}_{0} X$ and $g \in \mathbb{C}_{0}(Y)$ and we choose any points $x_{0} \in X, y_{0} \in Y$, then $(f, g)=V_{0}(h)$ where $h$ is the function in $\mathbb{C}_{0}(X \times Y)$ defined by

$$
h(x, y)=\delta_{x_{0}}(x) g(y)+f(x) \delta_{y_{0}}(y) .
$$

Let $\langle\cdot, \cdot\rangle$ be the sesquilinear form defined on $\mathbb{C}_{0}(X \times Y)$ by $c$ :

$$
\langle f, g\rangle=\sum f(x, y) \overline{g\left(x^{\prime}, y^{\prime}\right)} c\left((x, y),\left(x^{\prime}, y^{\prime}\right)\right),
$$

the sum extended over all $x, x^{\prime} \in X$ and all $y, y^{\prime} \in Y$. Using

$$
c\left((x, y),\left(x^{\prime}, y^{\prime}\right)\right)=a\left(x, x^{\prime}\right)+b\left(y, y^{\prime}\right),
$$

we find that

$$
\begin{aligned}
\langle f, g\rangle & =\sum_{x, x^{\prime}} f_{1}(x) \overline{g_{1}\left(x^{\prime}\right)} a\left(x, x^{\prime}\right)+\sum_{y, y^{\prime}} f_{2}(y) \overline{g_{2}\left(y^{\prime}\right)} b\left(y, y^{\prime}\right) \\
& =\left\langle f_{1}, g_{1}\right\rangle_{1}+\left\langle f_{2}, g_{2}\right\rangle_{2}
\end{aligned}
$$

where $\langle\cdot, \cdot\rangle_{1}$ and $\langle\cdot, \cdot\rangle_{2}$ are the sesquilinear forms defined on $\mathbb{C}_{0} X$ and $\mathbb{C}_{0} Y$ by $a$ and $b$ respectively. This formula implies that $V_{0}$ induces a surjective isometry of inner product spaces

$$
V: \mathbb{C}_{0}(X \times Y) / N \rightarrow \mathbb{C}_{0}(X) / N_{1} \oplus \mathbb{C}_{0} Y / N_{2}
$$

and so the closure of $V$ is a unitary operator from $H(X \times Y, c)$ to $H(X, a) \oplus H(Y, b)$.

THEOREM 4.4. For any two product systems $E, F$ we have

$$
\operatorname{dim}(E \otimes F)=\operatorname{dim} E+\operatorname{dim} F .
$$

Proof. Assume first that at least one of the two sets $\mathscr{U}_{E}, \mathscr{U}_{F}$ is void. Then at least one of the two cardinals $\operatorname{dim} E, \operatorname{dim} F$ is $c$, and hence their sum is $c$. On the other hand, Corollary 3.9 implies that $\mathscr{U}_{E \otimes F}$ must be void and so $\operatorname{dim}(E \otimes F)$ is also $c$. 
Thus we can assume that both $E$ and $F$ possess units. Let $\left(\mathscr{U}_{E}, a\right)$, $\left(\mathscr{U}_{F}, b\right)$, and $\left(\mathscr{U}_{E \otimes F}, c\right)$ be the covariance functions of $E, F$, and $E \otimes F$ respectively and let $\left(\mathscr{U}_{E} \times \mathscr{U}_{F}, d\right)$ be the sum of $\left(\mathscr{U}_{E}, a\right)$ and $\left(\mathscr{U}_{F}, b\right)$ :

$$
d\left((u, v),\left(u^{\prime}, v^{\prime}\right)\right)=a\left(u, u^{\prime}\right)+b\left(v, v^{\prime}\right) .
$$

We claim that

$$
\operatorname{dim} H\left(\mathscr{U}_{E} \times \mathscr{U}_{F}, d\right)=\operatorname{dim} H\left(\mathscr{U}_{E \otimes F}, c\right)=\operatorname{dim}(E \otimes F) .
$$

By Lemma 4.3(i), it is enough to observe that the map $\theta: \mathscr{U}_{E} \times \mathscr{U}_{F} \rightarrow$ $\mathscr{U}_{E \otimes F}$ defined by $\theta(u, v)=u \otimes v$ is surjective and satisfies

$$
c\left(\theta(u, v), \theta\left(u^{\prime}, v^{\prime}\right)\right)=a\left(u, u^{\prime}\right)+b\left(v, v^{\prime}\right) .
$$

The surjectivity of $\theta$ is immediate from (3.9). If $u, u^{\prime} \in \mathscr{U}_{E}$ and $v, v^{\prime} \in \mathscr{U}_{F}$ then for every $t>0$ we have

$$
\begin{aligned}
e^{t c\left(u \otimes v, u^{\prime} \otimes v^{\prime}\right)} & =\left\langle(u \otimes v)_{t},\left(u^{\prime} \otimes v^{\prime}\right)_{t}\right\rangle \\
& =\left\langle u_{t} \otimes v_{t}, u_{t}^{\prime} \otimes v_{t}^{\prime}\right\rangle=\left\langle u_{t}, u_{t}^{\prime}\right\rangle\left\langle v_{t}, v_{t}^{\prime}\right\rangle \\
& =e^{t a\left(u, u^{\prime}\right)} e^{t b\left(v, v^{\prime}\right)}=e^{t\left(a\left(u, u^{\prime}\right)+b\left(v, v^{\prime}\right)\right)},
\end{aligned}
$$

and (4.6) follows from this.

Finally, Lemma 4.3(ii) implies that

$$
\begin{aligned}
\operatorname{dim} H\left(\mathscr{U}_{E} \times \mathscr{U}_{F}, d\right) & =\operatorname{dim} H\left(\mathscr{U}_{E}, a\right)+\operatorname{dim} H\left(\mathscr{U}_{F}, b\right) \\
& =\operatorname{dim} E+\operatorname{dim} F,
\end{aligned}
$$

and we are done.

The main result on additivity of the index of $E_{0}$-semigroups is now a simple consequence of (4.4).

Corollary 4.7. Let $\alpha, \beta$ be $E_{0}$-semigroups. Then we have

$$
d_{*}(\alpha \otimes \beta)=d_{*}(\alpha)+d_{*}(\beta)
$$

Proof. Let $E_{\alpha}$ and $E_{\beta}$ be the product systems associated to $\alpha$ and $\beta$ as in $\S 2$ of [1]. By ([1], Proposition 3.15 et seq.), $E_{\alpha \otimes \beta}$ is isomorphic to the tensor product of product systems $E_{\alpha} \otimes E_{\beta}$. Hence

$$
d_{*}(\alpha \otimes \beta)=\operatorname{dim} E_{\alpha \otimes \beta}=\operatorname{dim}\left(E_{\alpha} \otimes E_{\beta}\right) .
$$

By Theorem 4.4 the right side is

$$
\operatorname{dim} E_{\alpha}+\operatorname{dim} E_{\beta}=d_{*}(\alpha)+d_{*}(\beta) .
$$




\section{REFERENCES}

[1] W. Arveson, Continuous analogues of Fock space, (to appear).

[2] R. T. Powers, An index theory for semigroups of endomorphisms of $B(H)$ and type II factors, Canad. J. Math., XL, No. 1 (1988), 86-114.

[3] , A non-spatial continuous semigroup of *-endomorphisms of $B(H)$, Publ. Res. Inst. Math. Sciences, Kyoto, (to appear).

[4] R. T. Powers and D. Robinson, An index for continuous semigroups of *-endomorphisms of $B(H)$, J. Funct. Anal. (to appear).

[5] V. S. Varadarajan, Geometry of Quantum Theory, II, Van Nostrand, New York, 1970.

Received March 15, 1988. Research supported in part by NSF Grant DMS-86-00375.

UNIVERSITY OF CALIFORNIA

BERKELEY, CA 94720 


\section{PACIFIC JOURNAL OF MATHEMATICS EDITORS}

\author{
V. S. VARADARAJAN \\ (Managing Editor) \\ University of California \\ Los Angeles, CA 90024 \\ Herbert Clemens \\ University of Utah \\ Salt Lake City, UT 84112 \\ THOMAS ENRIGHT \\ University of California, San Diego \\ La Jolla, CA 92093
}

R. FINN

Stanford University

Stanford, CA 94305

HeRmanN FlaschKa

University of Arizona

Tucson, AZ 85721

VAUGHAN F. R. JONES

University of California

Berkeley, CA 94720

STEVEN KeRCKHOFF

Stanford University

Stanford, CA 94305
RoBION KIRBY

University of California

Berkeley, CA 94720

C. C. MOore

University of California

Berkeley, CA 94720

HAROLD STARK

University of California, San La Jolla, CA 92093

\section{ASSOCIATE EDITORS}
R. ARENS
E. F. BECKENBACH
B. H. NeUmanN
F. WolF
K. YosHIDA (1906-1982)

\section{SUPPORTING INSTITUTIONS}
UNIVERSITY OF ARIZONA
UNIVERSITY OF BRITISH COLUMBIA CALIFORNIA INSTITUTE OF TECHNOLOGY UNIVERSITY OF CALIFORNIA MONTANA STATE UNIVERSITY UNIVERSITY OF NEVADA, RENO NEW MEXICO STATE UNIVERSITY OREGON STATE UNIVERSITY
UNIVERSITY OF OREGON UNIVERSITY OF SOUTHERN CALIFORNIA STANFORD UNIVERSITY UNIVERSITY OF HAWAII UNIVERSITY OF TOKYO UNIVERSITY OF UTAH WASHINGTON STATE UNIVERSITY UNIVERSITY OF WASHINGTON 


\section{Pacific Journal of Mathematics}

\section{Vol. 137, No. $1 \quad$ January, 1989}

V. S. Varadarajan, Henry Abel Dye $\ldots \ldots \ldots \ldots \ldots \ldots \ldots \ldots \ldots \ldots$ iii

Huzihiro Araki, An application of Dye's theorem on projection lattices to orthogonally decomposable isomorphisms $\ldots \ldots \ldots \ldots \ldots \ldots \ldots \ldots \ldots$

Richard Arens, The limit of a sequence of squares in an algebra need not be

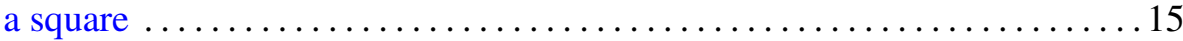

William Arveson, An addition formula for the index of semigroups of

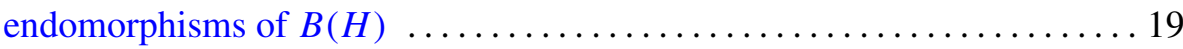

Robert James Blattner and Susan Montgomery, Crossed products and

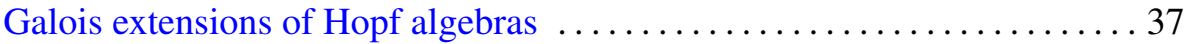

Erik Christensen and Allan M. Sinclair, On the vanishing of $H^{n}\left(\mathscr{A}, \mathscr{A}^{*}\right)$

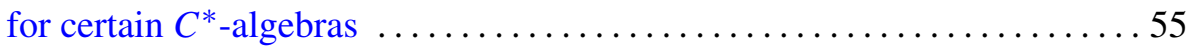

Philip C. Curtis, Jr. and Michael M. Neumann, Nonanalytic functional

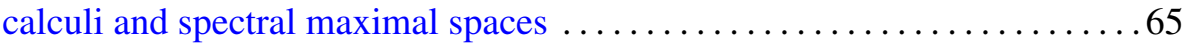

George A. Elliott and David E. Handelman, Addition of $C^{*}$-algebra extensions

Yaakov Friedman and Bernard Russo, Some affine geometric aspects of

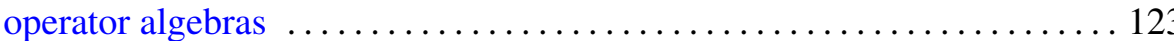

Valentin Ya. Golodets and Sergey D. Sinelshchikov, Regularization of actions of groups and groupoids on measured equivalence relations . . . 145

Irving Kaplansky, CCR-rings 155

Hideki Kosaki, Characterization of crossed product (properly infinite case)

Gert Kjærgaard Pedersen, Three quavers on unitary elements in $C^{*}$-algebras

Sorin Popa, Relative dimension, towers of projections and commuting squares of subfactors

Martin E. Walter, On a new method for defining the norm of

Fourier-Stieltjes algebras 